Researchers modify the Keap1/Nrf2 signaling system in Drosophila and demonstrate a survival benefit for those with more active signaling systems when exposed to toxins

\title{
Drosophila model offers potential way of boosting natural anti-oxidant response
}

The Keap1/N rf2 chemical signaling system has long been recognized in humans and constitutes one of the major ways that the body defends itself against toxic assaults and carcinogenic substances such as cigarette smoke. The KEAP1 gene senses danger and releases NRF2, which triggers rampant anti-oxidant activity in the cell.

The pathway has only recently been identified in D rosophila, potentially opening the door to faster, less expensive ways to identify compounds that stimulate natural anti-oxidant

activity. "This is one of the main mechanisms the body uses to fight off the things that give you cancer," states author Dirk Bohmann, a professor of Biomedical $G$ enetics at the University of Rochester (NY, USA). "T his puts cells into an antioxidant defense mode. D rug development and testing is very, very expensive and time-consuming. This work should speed the development of new drugs aimed at preventing cancer," he added.

The investigators discovered a gene called CN C in D rosophila. The CN C gene is widely known to be involved in determining the development of a fruit fly's head, and was found to serve like NRF 2, turning on cellular defenses on a broad scale. These cellular defenses include activation of thioredoxins and glutathione S-transferases, which are powerful anti-oxidants. Unlike many other anti-oxidants, whose effects in the body are transient, a fundamental genetic change such as a boost in N RF2 activity throughout an organism would supply an ongoing amplified anti-oxidant response, Bohman notes.

In these latest experiments, D rosophila were modified so they would glow green when exposed to ultraviolet light when the signaling pathway was functioning. Their results demonstrated that the $D$ rosophila with more active CN C glowed more brightly than the regular D rosophila, providing the team with an easy, visual way to identify whether the pathway is activated.

The team demonstrated the technology using a compound called oltipraz, which targets the Keap1/N rf2 pathway and has been tested in people as a cancerprevention agent. Those $D$ rosphila that ate food containing the compound glowed more strongly, demonstrating that the N RF2 pathway was more active in these flies.

W hen researchers boosted the activity of the pathway, fruit flies were three times morelikely to survive

W hile the main application of the work is in boosting the body's ability to resist cancer, the research could al so make a difference for patients who have cancer that is resistant to current drugs. In 2006, a team from Johns $\mathrm{H}$ opkins showed how this same signaling pathway allows some cancer cells to fight off drugs intended to kill them. G aining a foothold on the system in D rosophila would provide researchers with an additional tool as they search for ways to thwart these rogue cancer cells. an exposure to a toxin than regular flies. Furthermore, D rosophila with a more active signaling system lived $10 \%$ longer than the other flies. It is the first time that the system, long known to be an important anti-oxidant and cancerprevention pathway, has also been shown to play a role in providing an organism with a longer lifespan.

Source: Sykiotis GP, Bohmann D : Keap1/N rf2 signaling regulates oxidative stress tolerance and lifespan in D rosophila. D ev. Cell 14(1), 76-85 (2008) 


\section{Prio rity Paper Alerts}

Could ultraviolet B irradiance and vitamin D be associated with low er incidence rates of lung cancer?

M ohr SB, Garland CF, Gorham ED, Grant WB, Garland FC: J. Epidemiol. Community Health 62, 69-74 (2008).

Examines whether insufficient ultraviolet $B$ (UVB) irradiance, a marker of vitamin $D$ inadequacy, might contribute to lung cancer incidence. The study investigated the association of latitude and UVB irradiance with age-adjusted incidence rates of lung cancer in 111 countries. Multiple regression was used to assess independent associations with UVB irradiance, and other factors, such as cigarette smoking. Results demonstrated that latitude was positively related to incidence rates in both men and women $\left(R^{2}=0.55, p<0.01\right.$ and $R^{2}=0.36, p<0.01$, respectively). In men, cigarette consumption $(p<0.001)$ was positively related to lung cancer risk, while there was an inverse association with UVB irradiance $(p=0.003)$. There were positive associations with UVB absorbers. UVB irradiance $w$ as also inversely associated $w$ ith incidence rates in women $(p=0.0002)$, whereas cigarette consumption $(p<0.001)$ was positively associated. The $R^{2}$ for the model was $0.77(p<0.001)$.

\section{Trisomy represses Apc(Min)-mediated} tumours in mouse models of Down's syndrome.

Sussan TE, Yang A, Li F, Ostrowski MC, Reeves RH: Nature 451(7174), 73-75 (2008) Investigated the relationship between trisomy and the incidence of intestinal tumours utilizing mouse models of Down's syndrome (Trisomy 21) and of cancer. Apc(M in)-mediated tumor number was determined in aneuploid mouse models Ts65Dn, Ts1Rhr and M s1Rhr. Trisomy for orthologues of approximately half of the genes on chromosome 21 (Hsa21) in Ts65Dn mice or only 33 of these genes in Ts1Rhr mice resulted in a significant reduction in the number of intestinal tumors. However, in M slRhr models, segmental monosomy for the same 33 genes that are triplicated in Ts1Rhr resulted in an increased number of tumors. Further studies indicated that it was the Ets2 gene that contributed most to the dosage-sensitive effect on intestinal tumor number. Furthermore, when overexpressed, the action of Ets2 as a repressor differs from tumour suppression, which requires normal gene function to prevent cellular transformation. In conclusion, upregulation of Ets2 and, potentially, of other genes involved in this kind of protective effect may provide a prophylactic effect in all individuals, regardless of ploidy.

\section{Proteomic profiling: more accurate than traditional biomarkers in identifying liver cancer?}

As the incidence of liver cancer continues to grow, fueled, in large part, by rising rates of hepatitis $\mathrm{C}$ infections, the search for tests to help diagnose the disease at an earlier stage intensifies. Latest research led by investigators from Beth Israel $D$ eaconess M edical C enter (M A, USA) demonstrates that a novel mass-spectrometry-based form of proteomic profiling is more accurate than traditional biomarkers in distinguishing liver cancer patients from patients with hepatitis $C$ liver cirrhosis, particularly with regard to identifying patients with small, curable tumors.

“Proteomics represents a potentially powerful tool for the serologic recognition of protein profiles associated with cancer," explains co-senior author Towia Libermann. "Although this particular proteomics technology had al ready proven capable of identifying liver cancer in some limited studies, this was the first time that the technology was compared side-by-side with the clinical standard biomarker in a cohort of patients at risk for developing the disease," he adds.

Currently, the best hope for early detection is cancer biomarkers and the current biomarker for liver cancer in clinical use is alpha fetoprotein (AFP). H owever, as Libermann explains, the AFP biomarker has a number of shortcomings, "AFP not only fails to detect many early tumors, but it also lacks specificity. Consequently, elevated AFP levels could be indicators of not only cancer, but also of other liver diseases or even benign conditions, while on the other hand, many patients with small tumors will test negative for AFP."
The authors, therefore, decided to evaluate the sensitivity and specificity of surface enhanced laser desorption/ionization time-of-flight mass spectrometry (SELDI-TO F M S) for the detection of liver cancer and to compare its effectiveness with AFP.

'This was the first time that the technology was compared side-byside with the clinical standard biomarker in a cohort of patients at risk for developing the disease.'

Investigators examined serum samples from 92 patients including 51 patients with liver cirrhosis and 41 patients with liver cancer, and among the cancer patients, individuals with both large and small (less than $2 \mathrm{~cm}$ ) tumors. U sing SELDI-TO F M S, the authors were able to identify an 11-protein signature that accurately discriminated between the cirrhosis and cancer patients. The resulting diagnostic value $74 \%$ sensitivity and $88 \%$ specificity - compared favorably with the diagnostic accuracy of AFP $(73 \%$ sensitivity and $71 \%$ specificity) as well as with two other biomarkers currently in clinical development for liver cancer, AFP-L3 and PIVKA-IL.

"M ost strikingly," notes Libermann, "in patients with small tumors (less than $2 \mathrm{~cm}$ ), where AFP identified only three, and AFP-L3 and PIVKA-II only one each, the 11-protein signature correctly identified seven of eight patients at this early stage of disease. This study supports the notion that a combination of multiple biomarkers may prove more effectivethan individual biomarkers for diagnosis of liver cancer, as well as other cancers."

Source: www.biocompare.com 


\section{Evidence suggests that breast cancer risk varies among BRCA carriers}

According to research recently published in the Journal of American M edical A ssociation, the risk for developing breast cancer varies widely among women who carry the $B R C A 1$ and $B R C A 2$ gene mutations.

Researchers identified 181 breast cancer patients who were also carriers of a $B R C A 1$ or BRCA2 mutation and thereafter examined the incidence of breast cancer in their first-degree female relatives. Both age and contralateral breast cancer in proband women were found to be determinants of the risk for breast cancer in relatives.

While previous studies have reported on the overall increased breast cancer risk among carriers of the BRCA1 and BRCA2 mutations, little attention has been paid to the degree in which the risk may vary among carriers. U Itimately, the ability to determine an individual's risk is critical for guiding decisions concerning cancer prevention options.

In the present study, a total of 103 breast cancers were reported among the 598 first-degree female relatives of the breast cancer patients. Relatives of women diagnosed with breast cancer at 35 years of age or younger were more than twice as likely to develop breast cancer as the relatives of women diagnosed between the ages of 45 and 55 years.

Similarly, the relatives of women diagnosed with contralateral breast cancer were $40 \%$ more likely to develop breast cancer than the relatives of women with unilateral breast cancer.

The investigators state "Accurate estimation of the risks conferred in the population and identification of important sources of variation in these risks constitute important scientific goals with significant implications for the clinical management of female carriers of BRCA1 or BRCA2 mutations," concluding that "our results underscore the conclusion that there is no single risk associated with BRCA1 or BRCA2 carrier status."

Source: Begg CB, H aile RW, Borg A: Variation of breast cancer risk among BRCA1/2 carriers. JAM A 299(2), 194-201 (2008).

\section{Saliva test may prove beneficial in diagnosis of early non-invasive breast cancer}

A chemical test that quantifies specific protein markers in human saliva can be used to accurately detect breast cancer, according to American researchers.

The objective of the study was to determine if protein by-products, secondary to cancer-related oncogenes, were present in the saliva of breast cancer patients.

Researchers obtained saliva samples from 10 healthy women, 10 women with benign breast tumors, and 10 women diagnosed with ductal carcinoma in situ. Saliva from each of the pooled samples was trypsinized and peptide digests were labeled with an isotopic reagent. Labeled peptides werethen combined and analyzed by reverse phase capillary chromatography. Expression of isotopically tagged proteins was compared between both tumor groups and with the healthy control group.
"We go through a process that compares different samples by chemically labeling them in such a way that we can not only identify the protein, but determine how much of it is in each sample," states W illiam D ubinsky and colleagues from the $U$ niversity of Texas $H$ ealth Science C enter (TX, USA) adding "this allows us to compare the levels of 150-200 different proteins in cancerous versus non-cancerous specimens to identify possible markers for disease."

The results of the salivary analyses in this population of patients yielded approximately 130 proteins in the saliva specimens and a total of 49 of these proteins were differentially expressed between the healthy control pool and the benign and cancer patient groups.

Thestudy therefore suggests that saliva is afluid suffused with solubilized by-products of oncogenic expression and that these proteins may be modulated secondary to ductal carcinoma in stu. Additionally, there may be salivary protein profiles that are uniqueto both ductal carcinoma in situ and fibroadenoma tumors.

D ubinsky and colleagues say that the saliva test represents an early and noninvasive method of differentiating between benign tumors and breast cancer. They aim to ultimately bring the diagnostic test, which they claim is capable of detecting the presence of cancer beforetheformation of a tumor, into dental offices or healthcare facilities, thereby facilitating early detection and physician referral. Source: Streckfus CF, M ayorga-Wark O, Arreola D, Edwards C, Bigler L, Dubinsky W P: Breast cancer related proteins are present in saliva and are modulated secondary to ductal carcinoma in stu of the breast. Cancer Invest 26, 109-117 (2008)

\footnotetext{
About the Bulletin Board If you have newsworthy information, please contact:

Vicki Millen, Commissioning Editor, Future Oncology; Future Medicine Ltd, Unitec House, 2 Albert Place, London N3 1QB, UK Tel.: +44 (0)20 8371 6090; Fax: +44 (0)20 83432313 v.millen@futuremedicine.com
}

The Bulletin Board highlights some of the most important events and research in the field of oncology. 